\title{
4 interaction dynamics occurred in telephone counselling for smoking cessation
}

Bottorff JL, Johnson JL, Moffat B, et al. Synchronizing clinician engagement and client motivation in telephone counseling. Qual Health Res 2004; 14:462-77.

Q What are the optimal characteristics of proactive telephone support as a smoking cessation intervention?

DESIGN

Qualitative study within a randomised controlled trial.

\section{SETTING}

Vancouver, British Columbia, Canada.

\section{PARTICIPANTS}

A sample of 56 consecutively enrolled patients (mean age 50 y, 55\% women, mean 31 y smoking) from a surgical preassessment clinic who were in the intervention group of a randomised controlled trial evaluating proactive smoking cessation telephone counselling by 8 trained nurses. Patients were self identified smokers, had elective surgery scheduled within 12 weeks after assessment, and had an anticipated hospital stay $\geqslant 24$ hours after surgery.

\section{METHODS}

All telephone counselling intervention calls for the 56 postoperative patients were tape recorded ( 368 completed calls, mean $6.2 \mathrm{~min} /$ call, range $1-50 \mathrm{~min}$ ), with the number of calls per patient ranging from 2-9. The calls were transcribed verbatim and checked for accuracy. Data analysis was done using the constant comparative method.

\section{MAIN FINDINGS}

The interaction dynamics that occurred during telephone calls varied depending on the nurse's level of engagement and the participant's level of motivation to quit smoking. 4 interaction dynamics emerged: affirming/working, chasing/skirting, controlling/withdrawing, and avoiding commitment.

Affirming/working occurred when nurses were engaged and participants were motivated. Nurses affirmed participants' cessation efforts and used strategies such as reflective listening, active helping, supporting self efficacy, and empathetic responses. The participants were actively working towards cessation by openly sharing their thoughts and experiences and suggesting cessation strategies of their own.

Chasing/skirting occurred when nurses were engaged and participants were not motivated. Nurses made suggestions, attempted to develop a plan, and tried to chase specific commitments or promises for future efforts, whereas participants skirted the issue by being noncommittal, giving vague responses and weak promises, and trying to close down the conversation. Participants frequently downplayed the amount they smoked and changed the subject.

In the controlling/withdrawing dynamic, nurses dominated and controlled the conversation in an overenthusiastic and authoritative tone causing participants to withdraw from discussing smoking. Nurses overused assessment questions or gave excessive advice, often beginning with phrases such as "you should..." or "have you tried...?" Participants had few opportunities to interject and became overwhelmed and non-committal.

For correspondence: Dr J $\mathrm{Z}$ Bottorff, Nursing and Health Behaviour Research Unit, School of Nursing, University of British Columbia, Vancouver, British Columbia, Canada. bottorff@nursing.ubc.ca

Sources of funding: British Columbia Medical Services Foundation and National Cancer Institute of Canada.
In the avoiding commitment dynamic, a superficial discussion of smoking occurred, in which nurses tended to accept the smoking status of participants quickly, and participants gave brief, uninformative responses. Nurses seemed tentative, almost condoning participants' smoking behaviour, and retreated to "safe" topics of conversation. Participants were negative and fatalistic about their chances of quitting smoking.

Interaction dynamics changed over the course of the telephone calls. Positive shifts in dynamics could occur as the result of sharing new insights or approaches; negative shifts could occur when participants confessed to smoking relapses even after considerable time was spent in supporting cessation.

\section{CONCLUSION}

An affirming/working dynamic, in which the telephone counsellor was engaged and the participant was motivated, was the optimal component in proactive telephone support for smoking cessation.

Commentary

roactive telephone counselling (when the counsellor initiates the call) may be a successful, low cost strategy for reaching specifically targeted groups such as smokers having elective surgery. Bottorff et al described the interactive dynamics between nurse counsellors and postoperative patients. The most optimal of the 4 dynamics occurred when nurses were engaged as indicated by careful listening and response to patient cues, and when patients were motivated.

Focusing on the dynamics of the counsellor-patient process is an important and understudied aspect of the Transtheoretical model of health behaviour change noted in the study. Although the nature of this research precluded an analysis of where each patient was within the 5 stages of the model, the literature supports a view that staging affects patients' chances of success.

The Tobacco Use and Dependence Guideline Panel has stated that smoking is a chronic condition frequently requiring multiple attempts to quit and repeated interventions. Intratreatment social support and problem solving are 2 identified types of counselling found to be particularly effective as part of smoking cessation interventions. ${ }^{1}$ Both seem to be present in the affirming/working dynamic described in this study.

The Tobacco Use and Dependence Clinical Practice Guideline provided insufficient evidence to assess the effectiveness of proactive telephone counselling. However, the study by Bottorff et al makes a valuable contribution to our understanding of the nature of telephone support dynamics that may be most effective in supporting smoking cessations.

George Shuster, RN, DNSc College of Nursing, University of New Mexico Albuquerque, New Mexico, USA

1 Fiore MC, Bailey WC, Cohen SL, et al. Treating tobacco use and dependence. Clinical Practice Guideline. Rockville, MD: US Department of Health and Human Services. Public Health Service. June, 2000. hitp:// www.ncbi.nlm.nih.gov/books/

bv.fcgi? call = bv.View..ShowSection\&rid $=$ hstat2.chapter.7644 